\title{
Chromosomal damage and telomere length in peripheral blood lymphocytes of cancer patients
}

\author{
SONA VODENKOVA ${ }^{1-3}$, MICHAL KROUPA $^{2,4}$, ZDENKA POLIVKOVA $^{1}$, LUDOVIT MUSAK $^{5}$, \\ MILOSLAV AMBRUS ${ }^{6}$, MICHAELA SCHNEIDEROVA ${ }^{7}$, RENATA KOZEVNIKOVOVA ${ }^{8}$, LUDMILA VODICKOVA ${ }^{2-4}$, \\ SIVARAMAKRISHNA RACHAKONDA ${ }^{9}$, KARI HEMMINKI $^{4,10}$, RAJIV KUMAR ${ }^{9}$ and PAVEL VODICKA ${ }^{2-4}$ \\ ${ }^{1}$ Department of Medical Genetics, Third Faculty of Medicine, Charles University, Prague 100 00; \\ ${ }^{2}$ Department of Molecular Biology of Cancer, Institute of Experimental Medicine of The Czech Academy of Sciences, \\ Prague $14200 ;{ }^{3}$ Institute of Biology and Medical Genetics, First Faculty of Medicine, Charles University, Prague 128 00; \\ ${ }^{4}$ Biomedical Centre, Faculty of Medicine in Pilsen, Charles University, Pilsen 323 00, Czech Republic; ${ }^{5}$ Biomedical Center Martin, \\ Jessenius Faculty of Medicine, Comenius University in Bratislava, Martin 036 01, Slovakia; Departments of ${ }^{6}$ Radiology and \\ Oncology and ${ }^{7}$ Surgery, Faculty Hospital Kralovske Vinohrady, Prague 100 34; ${ }^{8}$ Department of Oncosurgery, Medicon, \\ Prague 140 00, Czech Republic; ${ }^{9}$ Division of Molecular Genetic Epidemiology; ${ }^{10}$ Division of Cancer Epidemiology, \\ German Cancer Research Center, Heidelberg 691 20, Germany
}

Received November 29, 2019; Accepted July 24, 2020

DOI: $10.3892 /$ or.2020.7774

\begin{abstract}
Accumulation of non-specific structural chromosomal aberrations (CAs) and telomere shortening contribute to genome instability, which constitutes as one of the hallmarks of cancer. CAs arise due to direct DNA damage or telomere shortening. CAs in peripheral blood lymphocytes (PBL), which are considered to be markers of exposure, have been previously reported to serve a role in the pathophysiology and progression of cancer through mechanisms that are
\end{abstract}

Correspondence to: Dr Sona Vodenkova or Dr Pavel Vodicka, Department of Molecular Biology of Cancer, Institute of Experimental Medicine of The Czech Academy of Sciences, Videnska 1083, Prague 142 00, Czech Republic

E-mail: sona.vodenkova@iem.cas.cz

E-mail: pavel.vodicka@iem.cas.cz

Abbreviations: ACs, aberrant cells; aOR, adjusted odds ratio; $\mathrm{BC}$, breast cancer; CART, classification and regression tree; CAs, chromosomal aberrations; CAtot, total chromosomal aberrations; $\mathrm{CI}$, confidence interval; CIN, chromosomal instability; CRC, colorectal cancer; CSAs, chromosome-type aberrations; CTAs, chromatid-type aberrations; Her2, ErbB2 (Her2/neu) receptors; HR, hazard ratio; LC, lung cancer; OR, odds ratio; OS, overall survival; PBL, peripheral blood lymphocytes; PCR, polymerase chain reaction; PRs, progesterone receptors; RFS, recurrence-free survival; rs, Spearman's Q; RTL, relative telomere length; TL, telomere length; TNM, Tumor-Node-Metastasis

Key words: structural chromosomal aberrations, telomere length, peripheral blood lymphocytes, breast cancer, colorectal cancer, lung cancer, cancer susceptibility, patient long-term survival poorly understood. In addition, the prognostic relevance of telomere length (TL) in patients with cancer remains to be elucidated. In the present study, CAs and TL in PBL isolated from patients with newly diagnosed cancer (151 breast, 96 colorectal, 90 lung) and 335 cancer-free control individuals were investigated. These results were then correlated with clinicopathological factors and follow-up data. The accumulation of CAs in PBL was observed with increased susceptibility to breast and lung cancer $(\mathrm{P}<0.0001)$, while individuals with longer TL were found to be at a higher risk of breast cancer $(\mathrm{P}<0.0001)$. Increased chromatid-type aberrations were also revealed to be associated with lower overall survival of patients with breast and colorectal cancers using a multivariate model. Compared with control individuals, no association was observed between TL and CAs or age in patients with cancer. In conclusion, the present study demonstrates the association between CAs/TL in PBL and the susceptibility, prognosis and survival of patients with breast, colorectal and lung cancer.

\section{Introduction}

Tumorigenesis is a multistep process that is driven by a number of genetic alterations, such as chromosomal rearrangements, which result in selective advantages during the process of cellular transformation, tumor development and progression $(1,2)$. The majority of cancers also undergoes substantial epigenetic alterations that alter the expression profile of critical genes during the malignant transformation process (3).

Acquired non-specific structural chromosomal aberrations (CAs) describe a group of multiple and diverse intra- or inter-chromosomal rearrangements. Structural CAs can be of either chromatid-type (CTAs) or chromosome-type (CSAs) depending on the stage of cell cycle and mechanism of clastogenic compounds involved. They arise as a consequence of either direct mutagenic effects and/or dysfunctions in 
DNA repair during the entire lifetime of an individual (4-6). Measurement of CAs in peripheral blood lymphocytes (PBL) is conventionally used for monitoring environmental and occupational exposure to clastogens $(7,8)$. Several European prospective and retrospective studies have previously demonstrated that increased frequencies of CAs in PBL can be applied as predictive markers for increased cancer susceptibility (9-12).

A number of CAs can occur as the primary events during cancer initiation, following which disease progression drives additional chromosomal changes, which can be used to reflect prognosis and cancer progression (13). Compared with specific chromosomal rearrangements that occur in tumor tissues during carcinogenesis, non-specific structural CAs in PBL can serve as a marker of cancer susceptibility. This hypothesis, which was summarized by Boffetta et al (14), was based on the presumption that genetic damage in PBL is reflective of changes in cells undergoing malignant transformation.

The formation of CAs has also been previously associated with the process of telomere shortening (15-17). Telomeres are unique chromatin structures at the ends of linear eukaryotic chromosomes characterized by tandem repeats (TTAGGG) and associated proteins $(18,19)$. Telomeres progressively shorten due to limitations in DNA synthesis after successive cycles of mitosis (20). Excessive telomere shortening can lead to proliferation arrest and cellular apoptosis. Additionally, loss of telomeres can result in a telomere crisis, causing genome instability leading to structural CAs (21). Therefore, telomere length (TL) serves a critical role in the maintenance of genome integrity and cancer initiation/progression.

In the present study, the potential association between acquired non-specific structural CAs and TL in the PBL of patients with a sporadic form of the breast (BC), colorectal (CRC) and lung cancer (LC) and control individuals was investigated. In addition, the potential prognostic relevance of CAs and TL was also addressed.

\section{Materials and methods}

Study population. The present study was performed by pooling data from cytogenetic studies performed between January 2006 and May 2013. The study population consisted of three groups of newly diagnosed and histologically confirmed individuals with BC $(\mathrm{n}=151)$, CRC $(\mathrm{n}=96)$ and LC $(n=90)$, along with a group of control individuals $(n=335)$. Patients were recruited at the 3rd Faculty of Medicine, Charles University (Prague, Czech Republic) and the Jessenius Faculty of Medicine, Comenius University and University Hospital (Martin, Slovakia).

Control individuals who were matched for both age and sex, including 145 women for comparison with women with BC, were recruited at the Blood Centre of Faculty Hospital Kralovske Vinohrady (Prague, Czech Republic) and from the Department of Surgery, General University Hospital (Prague, Czech Republic).

The present study adhered to the ethical guidelines of the Helsinki Declaration. All participants were informed about all aspects of the study, agreed with the purpose of the study and procedures to be undertaken, following which they provided informed consent. The present study design was approved by the local Ethics Committees of all participating hospitals.
Samples and data collection. Blood samples $(2 \times 5 \mathrm{ml})$ were obtained from control individuals and patients with cancer. Fresh blood samples treated with heparin were used immediately for cytogenetic chromosomal analysis after transportation at $4^{\circ} \mathrm{C}$ whereas blood samples treated with EDTA were frozen at $-20^{\circ} \mathrm{C}$ for subsequent DNA isolation and TL measurement.

Information regarding patient characteristics, including demographics, family history of cancer, smoking habit, occupational history, body mass index and the presence of other diseases, including hypertension, diabetes mellitus, cardiovascular disease, along with their treatment regimen, were collected at the time of diagnosis using a structured questionnaire. Information on disease characteristics, including tumor location, tumor-node-metastasis (TNM) stage, histopathological grade, histological classification and the presence of hormonal receptors and administration of hormonal therapy in cases of BC were all collected following surgical resection. The characteristics of the study population were partially described previously by Vodenkova et al (12). Detailed data of the subjects in the present study are provided in the Table SI. The last follow-up date of the patients in the present study was July 2017.

The exclusion criteria for the recruitment of cancer patients in the present study were as follows: i) Personal history of previous malignancies; ii) hereditary forms of cancer; iii) prior use of radiotherapy or chemotherapy, to avoid the presence of additional chromosomal damage related to the treatment; and iv) prior use of X-rays, such as those used for diagnostic purposes. In addition to data from questionnaires and medical reports, the last criterion was also verified by comparing the frequencies of typical post-radiation aberrations including dicentric and ring chromosomes, in the study population. The frequencies of these CAs did not differ between the patients with cancer and corresponding controls (12). For survival analyses, the following additional exclusion criteria were applied for patients: i) Those who had either missing data regarding their living status $<5$ years; ii) underwent a 2 nd surgery for unknown reasons; or iii) had experienced duplicate tumors. Consequently, 4 patients with $\mathrm{LC}$ were disqualified from the final analysis. The entire data set comprised 208 patients who were still alive and 125 patients who were deceased as a result of their respective cancers.

Chromosomal analysis. Levels of structural CAs in PBL were evaluated using the standard cytogenetic method as previously described by Vodicka et al (11). Briefly, blood cultivation was performed in complete medium (Chromosome medium P; cat. no. EKAMTB-100; EuroClone S.p.A.) for $50 \mathrm{~h}$ at $37^{\circ} \mathrm{C}$. After $48 \mathrm{~h}$ cultivation, cell division was stopped using colchicine (Sigma-Aldrich; Merck KGaA) during the first metaphase of mitosis. After a cytogenetic procedure, microscopic slides were stained conventionally using Giemsa (Sigma-Aldrich; Merck KGaA). Microscopical analysis of 100 metaphases with $46 \pm 1$ chromosomes was performed blindly by two independent scorers for each sample. The percentages of aberrant cells (ACs), total chromosomal aberrations (CAtot), CTAs (chromatid breaks and exchanges) and CSAs (chromosome breaks, terminal and interstitial deletions, dicentric and ring chromosomes with their difragments and abnormal chromosomes) were calculated. Representative microscopic images of chromosomes with different types of CAs are presented in Fig. S1. 
Standardization procedure has been applied for scoring CAs in the former Czechoslovakia and later in both Czech Republic and Slovakia (22). To minimize inter-laboratory and inter-scorer differences, random exchanges of microscope slides were performed between the two laboratories in Prague and Martin as a part of the standard operating procedure.

Measurement of TL. TL was measured as relative telomere length (RTL) using monochrome multiplex real-time qPCR assay as previously described by Cawthon (23) with slight modifications (24-26). All reactions were performed in triplicates in an optical 384-well reaction plate. Real-time PCR experiments were performed on a Viia 7 Real-time PCR System (Applied Biosystems; Thermo Fisher Scientific, Inc.) using two simultaneous programs to acquire the respective cycle threshold values for the telomere sequences and albumin gene. RTL was expressed as the ratio between telomere (T) and albumin (S; single-copy gene), which is also known as the $\mathrm{T} / \mathrm{S}$ ratio. Examples of the results from RTL measurements are presented in Fig. S2.

Statistical analysis. Statistical analysis of the data was performed using the SAS 9.4 software (SAS Institute Inc.). The results were processed into graphs using Statistica 12 (StatSoft, Inc.). P $<0.05$ was considered to indicate a statistically significant difference. Multiple pairwise comparisons were corrected using the Bonferroni method $(\mathrm{P}=0.025)$. Descriptive statistical analysis was conducted for the measured parameters on the whole data set in addition to individual groups.

Differences between controls and patients were tested using the median two-sample test or $\chi^{2}$-test. Association between the examined variables were tested using Spearman's rank correlation coefficient, which is expressed by Spearman's $\rho$ value $\left(r_{s}\right)$ and graphically plotted using linear regression. Subjects were stratified into two groups based on the frequency of CAs and TL using the median values measured for control individuals. The effect on cancer risk was determined by logistic regression and calculated by estimating the odds ratios (ORs) with the $95 \%$ confidence intervals (CIs). ORs were adjusted for age and sex (aORs). Only age was used for the adjustment of ORs for patients with BC, since all corresponding controls were female.

Clinical outcomes were evaluated by calculating patients' 5-year overall survival (OS) and recurrence-free survival (RFS). OS was defined as the time from the date of diagnosis to the date of death, which would then be marked as a negative event or the last follow-up (July 2017), which would be marked as censored data. RFS was calculated from the date of diagnosis to the date of local recurrence, distant metastasis discovery or death, which would then be marked as a negative event, or last follow-up (patients without any event) which is defined as censored data. The relative risk of mortality or recurrence was estimated using the hazard ratio (HR) with 95\% CIs using Cox regression. Multivariate survival analysis, referred to as a classification and regression tree (CART) (27), was performed using the Cox regression model to identify the most prognostically significant interactions between the factors investigated and the OS and RFS of the patients. Covariates used in the CART were baseline and clinicopathological factors alongside all types of CAs and TL.

\section{Results}

Case-control study. Levels of structural CAs, including ACs, CAtot, CTAs and CSAs, were found to be significantly higher in patients with BC and LC compared with those in the corresponding control groups. Following multiple testing corrections, no increased chromosomal damage was observed in patients with CRC compared with that in the control individuals (Table I). Individuals with higher frequencies of chromosomal damage were revealed to be at a higher risk of $\mathrm{BC}$ (ACs, aOR=4.81, $\mathrm{P}<0.0001$; CAtot, $\mathrm{aOR}=4.49, \mathrm{P}<0.0001$; CTAs, aOR=2.43, $\mathrm{P}=0.0004$; $\mathrm{CSAs}, \mathrm{aOR}=2.31, \mathrm{P}=0.0008)$ and LC (ACs, aOR=3.82, $\mathrm{P}<0.0001$; CAtot, $\mathrm{aOR}=3.44, \mathrm{P}<0.0001$; CTAs, aOR=3.30, $\mathrm{P}<0.0001)$. Although no association between CAs and CRC risk was found in the present set of patients, both age and sex were revealed to be significantly associated with the risk of CRC and LC (Table II).

Patients with BC exhibited significantly longer TL compared with control individuals $(\mathrm{P}<0.0001$; Table I), where longer TL was associated with an increased risk of $\mathrm{BC}$ $(\mathrm{aOR}=6.49 ; \mathrm{P}<0.0001$; Table II $)$. By contrast, patients with CRC and LC demonstrated moderately shorter TL compared with that in control individuals (Table I), which in turn was not associated with the risk of either types of cancer (Table II). A negative correlation was observed between the different types of $\mathrm{CA}$ and $\mathrm{TL}$ in individuals in the control group (ACs, $r_{s}=-0.21, P=0.0004$; CAtot, $r_{s}=-0.22, P=0.0003$; CTAs, $r_{s}=-0.10, P=0.10 ;$ CSAs, $r_{s}=-0.22, P=0.0002$; Fig. S3A). In addition, a significant correlation was also recorded between TL and age $\left(\mathrm{r}_{\mathrm{s}}=-0.62 ; \mathrm{P}<0.0001\right)$ and between CSAs and age $\left(r_{s}=0.17 ; \mathrm{P}=0.001\right)$ in control individuals. Associations between all types of CAs tested and TL (Fig. S3B-D) or between all the studied parameters and age (data not shown) in patients with cancer were not detected, except for in patients with LC, who exhibited a significant correlation between $\mathrm{ACs} / \mathrm{CAtot} / \mathrm{CSAs}$ and age $\left(\mathrm{AC}, \mathrm{r}_{\mathrm{s}}=0.23, \mathrm{P}=0.03\right.$; CAtot, $\mathrm{r}_{\mathrm{s}}=0.22, \mathrm{P}=0.04 ; \mathrm{CSAs}$, $\left.r_{s}=0.22, P=0.04\right)$.

Survival analyses. Significant differences were observed in both OS and RFS among patients with BC, CRC and LC $(\mathrm{P}<0.0001$, data not shown). After 5 years, the alive/dead proportion of patients with BC was 134/17 (88.7\%), 51/45 (53.1\%) for patients with CRC and 23/63 (26.7\%) for patients with LC. The RFS of patients was also found to significantly differ by cancer type $(\mathrm{P}<0.0001$, data not shown). For BC, 122 patients survived but 29 patients experienced an event during the follow-up period. Patients with CRC were notably more likely to experience an event and therefore demonstrated reduced RFS, with 36 patients surviving asymptomatically, events occurring in 60 patients. In patients with LC, the RFS was almost identical to that in OS, with 20 patients showing asymptomatic survival and 66 afflicted with event occurrence. As a result, each of the individual cancer groups were analyzed separately.

Univariate survival analyses. All data from the univariate analysis of the impact of well-established prognostic factors such as baseline and disease characteristics in addition to the CAs, including ACs, CAtot, CTAs, CSAs, and TL on the OS and RFS of patients were generated using the Cox regression hazard 


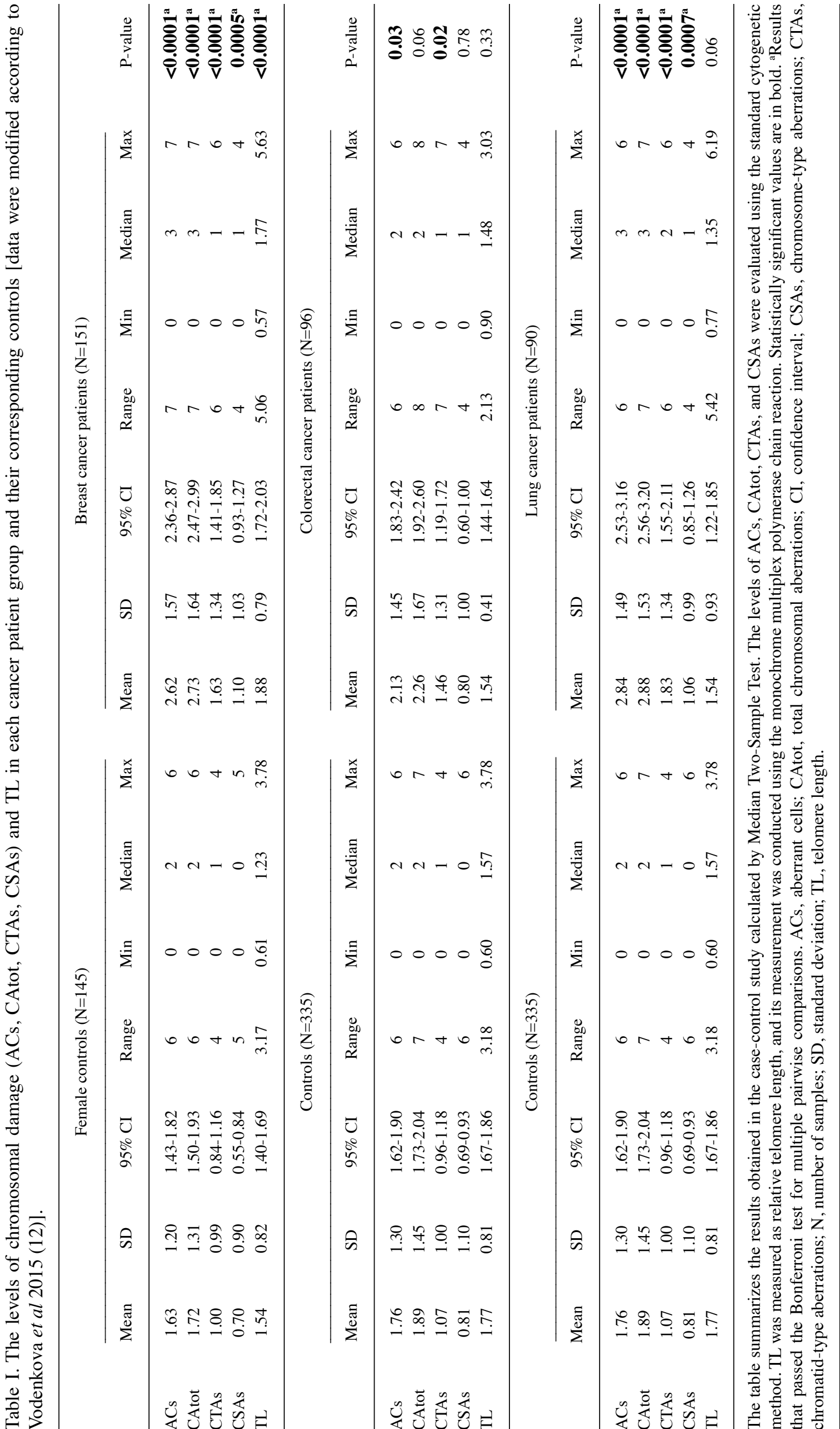




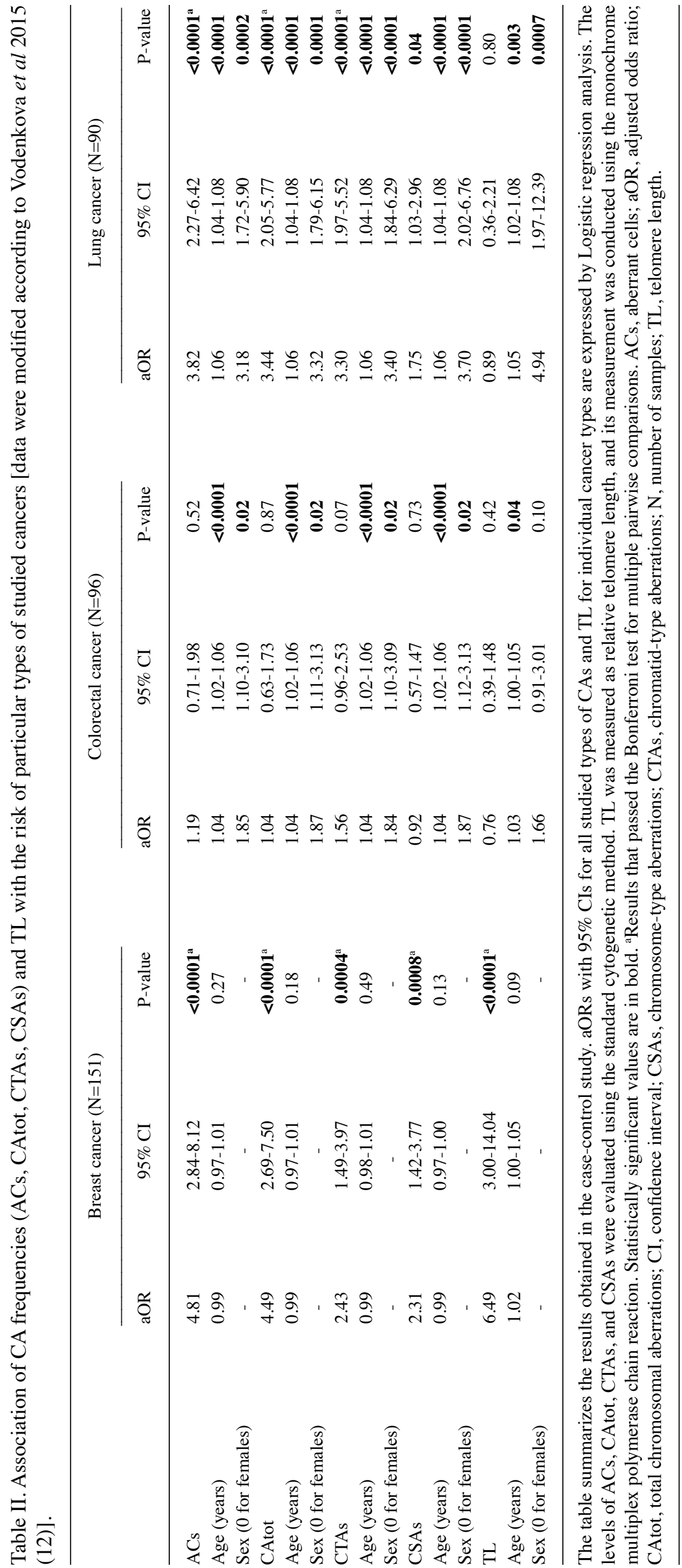




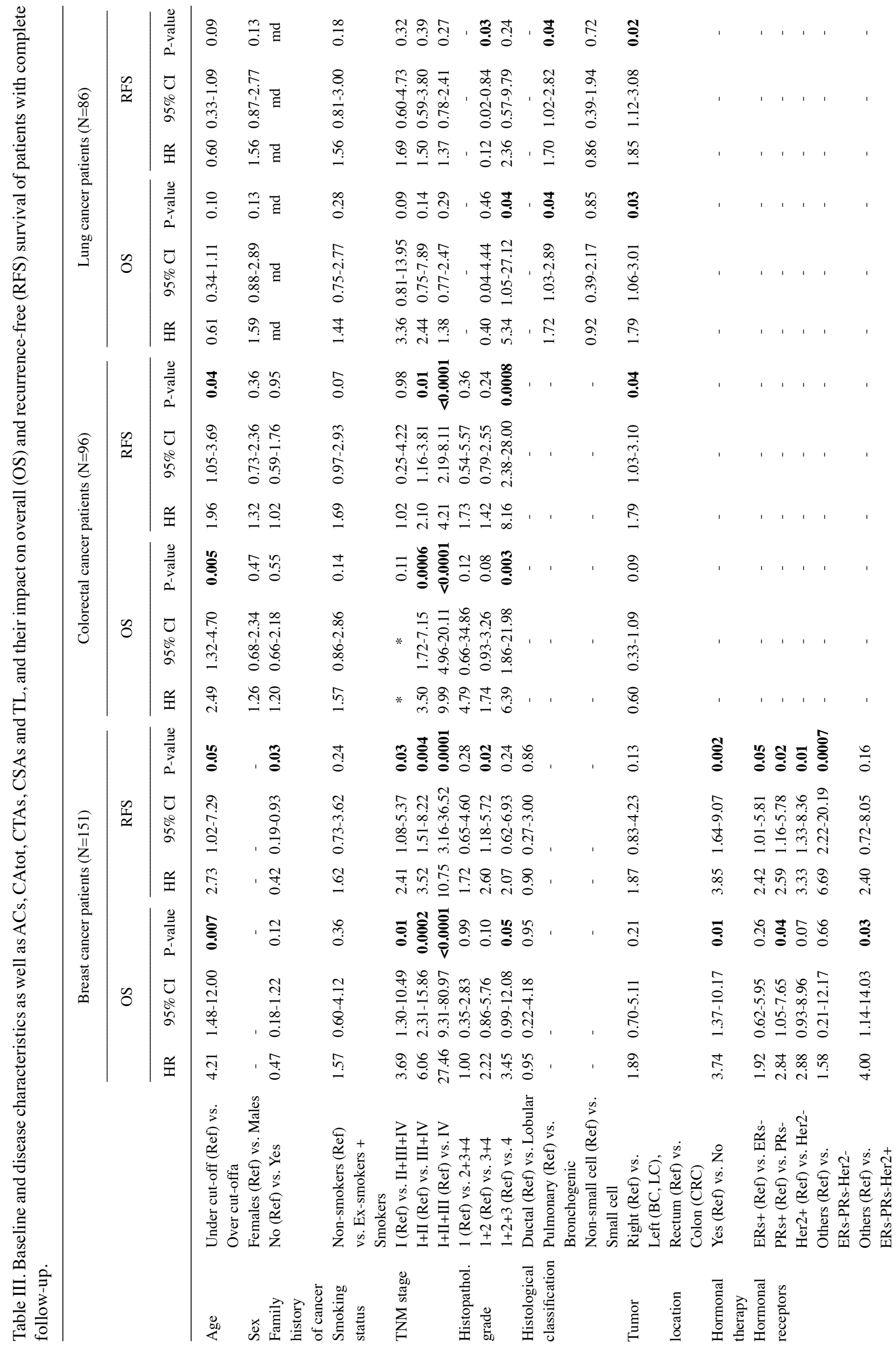




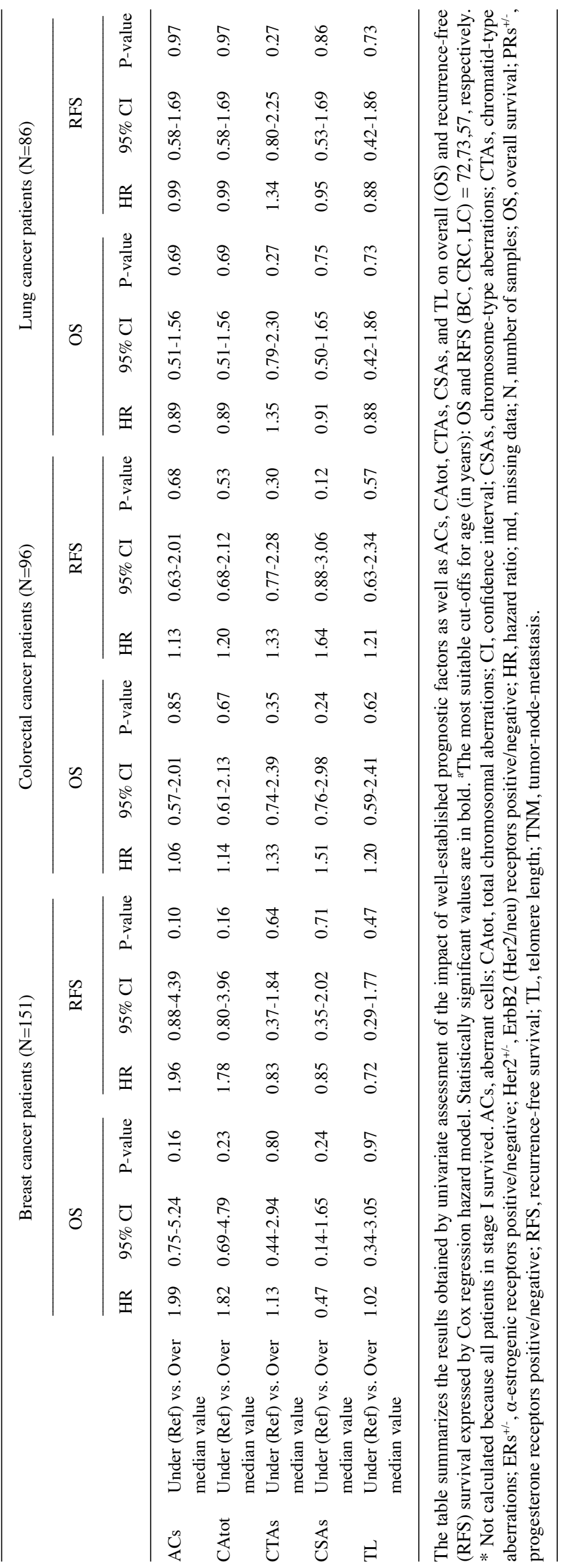


model and are presented in Table III. TNM stage, histopathological grade, histological classification and tumor location were found to modulate OS and RFS differently. Advancing age was found to be associated with lower OS and RFS in patients with $\mathrm{BC}$ and $\mathrm{CRC}$. In patients with $\mathrm{BC}$, the absence of both hormonal receptors and hormonal therapy was associated with lower OS and RFS. CAs and TL were not revealed to significantly associate with OS and RFS in patients with any cancer.

Multivariate survival analyses. To assess the prognostic utility of all the variables, the interactive effects of all studied types of CAs and TL together in association with baseline and disease characteristics were explored using a CART analysis. Covariates used in the CART were baseline and clinicopathological factors, specifically age, sex, family history of cancer, smoking status, TNM stage, histopathological grade, histological classification, tumor location, type of subsequent therapy received and the presence of hormonal receptors in breast tumors, in addition to all types of CAs and TL. The first split at the top of the tree denote the prognostic factor with the highest statistical significance. TNM stage was found to be the first parameter to split from the tree for predicting both OS (Fig. 1) and RFS (Fig. 2) in patients with $\mathrm{BC}$ and $\mathrm{CRC}$, whereas the laterality of tumor was found to be the point of first split in patients with LC.

In patients with $\mathrm{BC}$, additional factors apart from TNM stage were also revealed to serve key prognostic roles in predicting both OS and RFS. Among the variables found to be key determinants of the OS tree structure in patients with $\mathrm{BC}$ were progesterone receptors (PRs), histopathological grade, age and CTAs (Fig. 1A). Among TNM stages $0+\mathrm{I}$ + II + III, the next split revealed an interaction between PR positivity (positive vs. negative, 94.9 vs. 85.4\%) and histopathological grade (grades $1+2$ vs. grades $3+4,98.5$ vs. $86.7 \%$ ). Subsequently, patients with grade $1+2 \mathrm{BC}$ would display better OS if they were aged $>48$ years $(>48$ years vs. $<48$ years, 100 vs. $90 \%$ ). By contrast, patients with grade 3+4 BC would exhibit superior OS provided their levels of CTAs were $<2$ (0-2 vs. 3-7, 95 vs. $70 \%$ ). In terms of RFS, patients with BC were subsequently divided into two categories after the initial split - TNM stage $0+$ I + II and TNM III + IV (Fig. 2A). In the TNM $0+$ I + II group, the subsequent split was again found to be PR positivity/negativity (positive vs. negative, 94.3 vs. $65.1 \%$ ), which interacted with human epidermal growth factor receptor 2 (Her2/neu or ErbB2) positivity/negativity. The prognosis of patients with BC negative for PRs was improved by the presence of Her2 receptors (positive vs. negative, 87.5 vs. $50 \%$ ). Similarly, this was the case in patients with BC positive for PRs, where the next most statistically significant factor for predicted RFS was the presence of Her2 receptors (positive vs. negative, 100 vs. 88.8\%). Following stratification for PR positivity, RFS of patients with BC negative for Her2 was then found to be influenced by age ( $<64$ vs. $>64$ years, 90.8 vs. 83.3\%). In addition, patients with BC aged $<64$ years demonstrated CSA frequency as the terminal node of the CART analysis for RFS. Patients with BC without observable CSAs displayed worse prognosis (0 vs. 1-4, 72.7 vs. 100\%).

The only significant split found after the TNM stage was CTA frequency in patients with CRC (Fig. 1B). Patients with CRC in TNM stage III would have dramatically increased OS if they exhibit CTA levels $<1$ (0-1 vs. 2-7, 84.6 vs. $31 \%$ ). The only signifi- cant prognostic factor connected with RFS appeared to be the TNM stage in patients with CRC in the present study (Fig. 2B).

The OS of patients with LC was found to be associated with tumor laterality and histological classification (Fig. 1C), where patients with left-sided LC of the pulmonary type were more likely to survive after 5 years compared with patients with LC of the bronchogenic type (pulmonary vs. bronchogenic, 24.5 vs. $0 \%$ ). Similarly to OS, patients with left-sided LC of pulmonary histology also exhibited superior RFS (pulmonary vs. bronchogenic, 24.5 vs. 0\%; Fig. 2C). Additionally, patients with right-sided LC demonstrated an age-dependent association with prognosis.

\section{Discussion}

Both accumulations of chromosomal damage and telomere shortening contribute to genome and chromosomal instability (CIN) (28-30). The primary aim of the present study was to investigate the relationship between the quantity of particular types of chromosomal damage, including aberrant cells (ACs), total chromosomal aberrations (CAtot), chromatid-type aberrations (CTAs) and chromosome-type aberrations (CSAs), and telomere length (TL) in the peripheral blood lymphocytes (PBL) of patients with newly diagnosed cancer and corresponding control individuals. The results were analyzed alongside the baseline, clinicopathological and follow-up data. The impact of increased CAs and shorter TL on the risk of the most common cancers breast cancer (BC), colorectal cancer (CRC) and (lung cancer) LC was also investigated. To the best of our knowledge, no previous study has addressed the association between the frequencies of CAs and TL in the PBL of both patients with cancer and control individuals. The present study also examined the possible prognostic utility of these aforementioned biomarkers in assessing the 5-year OS and RFS of patients with BC, CRC and LC.

The present study successively concludes the series of long-term cytogenetic studies that were launched in 2006, which initiated with the collection of samples from patients with cancer and control individuals. Initially, in the first retrospective case-control study, the frequencies of chromosomal damage were first measured in 300 newly diagnosed patients with cancer matched with 300 control individuals (11). In that previous study, the group of patients was mainly comprised of those with breast, colorectal, prostate, uterus + ovary, head + neck and bladder + kidney cancers. Comparing the entire group of patients with cancer with control individuals, significant differences in the CA frequencies were found. However, following the classification of each individual patient by tumor type, only patients with certain types of solid tumors differed compared with controls in terms of $\mathrm{CA}$ frequencies. Therefore, the present study extended the number of patients by focusing on the cancer types with the highest rates of prevalence, namely $\mathrm{BC}, \mathrm{CRC}$ and LC. In a previous study, increased levels of chromosomal damage markers were reported in patients with $\mathrm{BC}, \mathrm{CRC}$ and LC compared with control individuals (12). In the present study, a complex regression analysis was performed to determine the predictive power of each type of CAs. Although statistically significant aORs were found for particular types of CAs in patients with cancer compared with those in control individuals, only higher frequencies of ACs and CAtot were found to influence the risk of BC and LC whereas 

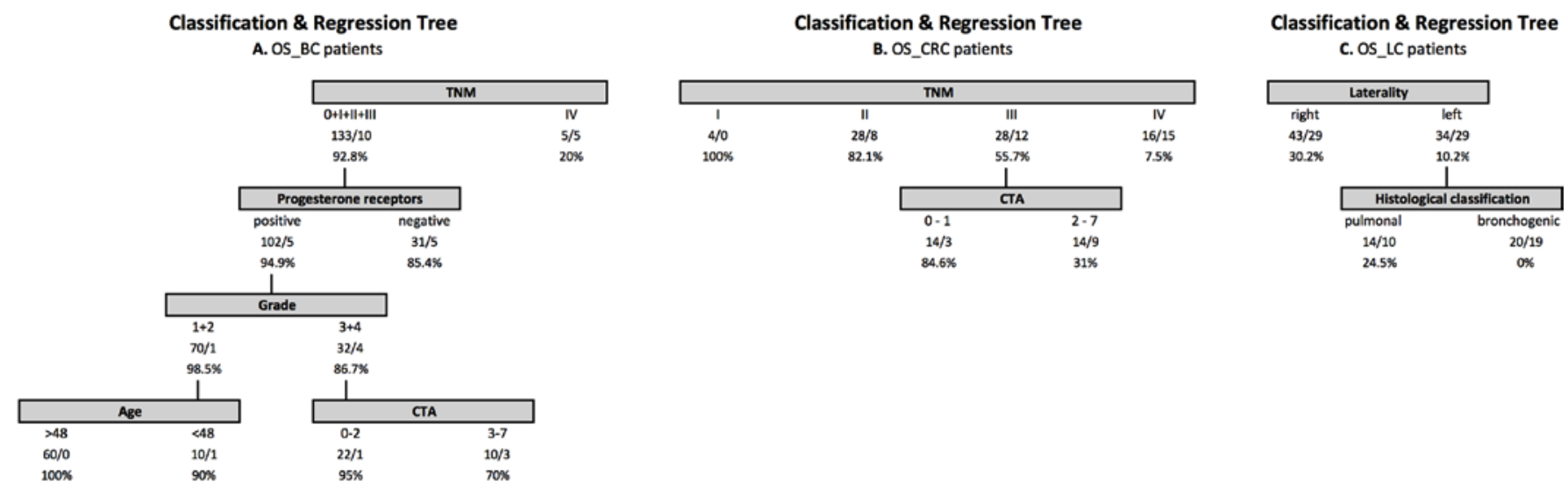

Figure 1. OS classification and regression trees. Classification and regression trees represent the results of multivariate survival analyses of patients with (A) BC, (B) CRC and (C) LC, generated using the Cox regression hazard model. Numbers under each node represent the total number of cases in a particular subcategory/number of events. Percentages below indicate the proportion of patients who survived after 5 years. BC, breast cancer; CRC, colorectal cancer; CTAs, chromatid-type aberrations; LC, lung cancer patients; OS, overall survival; TNM, tumor-node-metastasis.
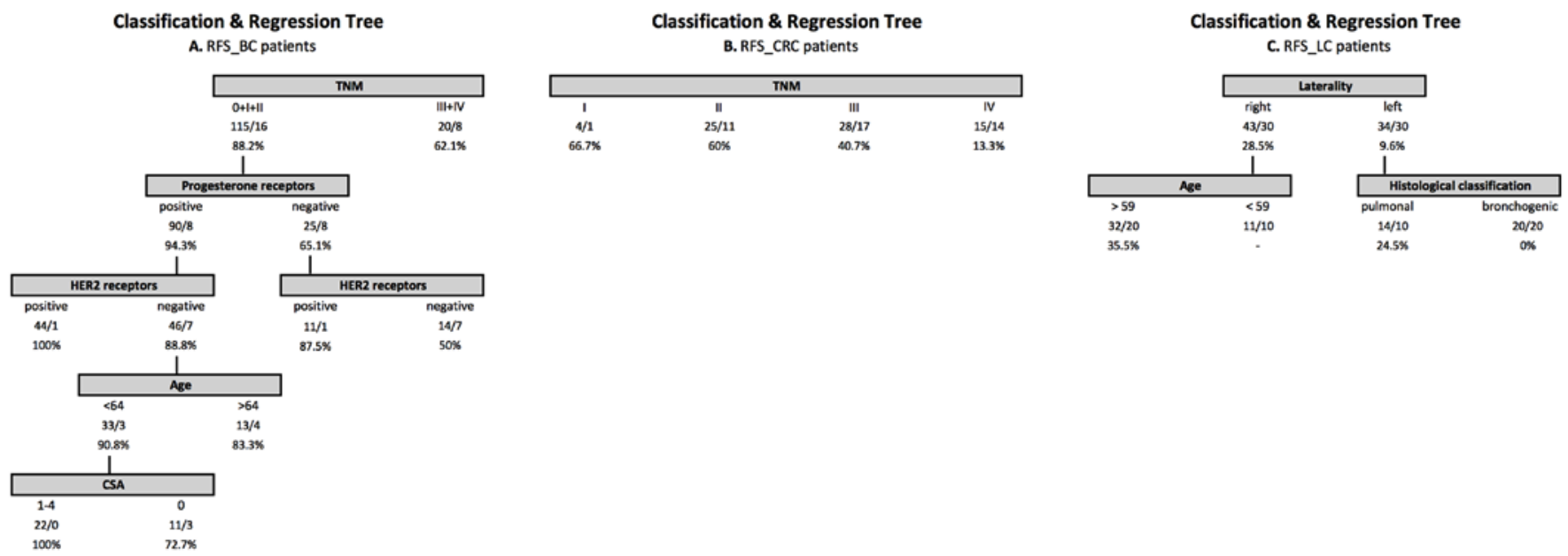

Figure 2. RFS classification and regression trees. Classification and regression trees represent the results of multivariate survival analysis of patients with (A) BC, (B) CRC and (C) LC, generated using the Cox regression hazard model. Numbers under each node represent the total number of cases in a particular subcategory/number of events. Percentages below indicate the proportion of patients who survived after 5 years. BC, breast cancer; CRC, colorectal cancer; CSAs, chromosome-type aberrations; LC, lung cancer; RFS, recurrence-free survival; TNM, tumor-node-metastasis.

and CTAs were found to influence the risk of LC from a clinical standpoint. Specifically, patients with elevated ACs and CAtot frequencies were at a higher risk of BC by 48 and $45 \%$, respectively and 38 and $34 \%$ for LC, respectively. In addition, higher quantities of CTAs were found to increase the risk of LC by $33 \%$. These observations confirmed the findings from previous studies, which were summarized by Bonassi et al (10). A cohort study conducted in Central Europe previously suggested that CSAs rather than CTAs are more accurate predictors of cancer risk (14). However, a pooled analysis involving $>1,000$ control individuals revealed an equally strong predictive power of both CTAs and CSAs for cancer (9).

Bonassi et al (10), did not find any of the covariates tested, specifically sex, smoking habit, age, time from the test and occupational exposure, to be potential modifiers of the effect of CA levels on the overall cancer risk in the whole cohort. In previous studies $(11,12)$, an effect of age and smoking on cancer risk was found, where these factors were employed to adjust the effect of CAs on BC, CRC and LC risk. The difference in the role of potential effect modifiers could be due to the difference in studied populations. Bonassi et al (10) conducted a study on cancer-free subjects who underwent CA screening for biomonitoring purposes, while our research studied groups consisting of newly diagnosed cancer patients. Cancer incidence is frequently dependent on age which, along with TNM stage, are well-established prognostic factors. However, data on CAs in the present study could be biased by the differences in stage/grade of the tumor and other clinicopathological characteristics at the time of diagnosis.

Measurement of CA frequencies in patients with cancer and healthy individuals was supplemented by TL determination in the present study. Telomeres are nucleoprotein structures that protect chromosomal ends from exonucleolytic degradation and the inappropriate activation of DNA repair pathways, which frequently results in the end-to-end fusion of non-homologous chromosomes (31). Li et al (15) and Xu et al (16), provided evidence that CAs may arise as a consequence of telomere dysfunction and the mechanisms associated with telomere shortening instead of direct DNA damage per se. Additionally, sub-telomeric regions appear to be hotspots for the formation 
of symmetrical exchanges between homologous chromatids. Cryptic aberrations in these regions have been previously shown to be associated with a number of human congenital abnormalities (4).

Comparing the TL between individuals in cancer and control groups, patients with $\mathrm{BC}$ were found to exhibit significantly longer TL compared with control individuals. In addition, patients with $\mathrm{BC}$ exhibited the longest TL of all groups investigated in the present study, including individuals in the CRC, LC and control groups. This observation may be explained by the fact that females typically harbor longer telomeres compared with males (32), where the set of patients with $\mathrm{BC}$ in the present study are comprised of only women. A number of hypotheses have been postulated to clarify this association, one of which is the presence of estrogen (33). An estrogen-responsive element has been previously demonstrated in telomerase reverse transcriptase ( $h T E R T)$, a catalytic subunit of the enzyme telomerase (34). Telomerase is responsible for extending the telomeric DNA at the very tips of linear chromosomes, thereby preventing the loss of genetic material in proliferating cells (18). Estrogen is also known to stimulate telomerase activity in breast cancer cells (35) where a functional estrogen response element has been identified in the proximal promoter of the hTERT gene. Therefore, direct estrogen effects have been considered to be a part of the telomerase induction mechanism (36). Supporting this, overexpression of estrogen has been reported to be one of the typical features of breast tumors and risk factors for BC.

The present set of patients with $\mathrm{BC}$ with longer telomeres were found with a $65 \%$ increased risk of BC. However, previous prospective and retrospective studies both showed that the association between TL in PBL and the risk of BC remains controversial. Several studies found an elevated risk among women with longer telomeres $(37,38)$ whereas other studies showed short telomere lengths to be associated with increased risk of BC (39-41). In addition, a number of previous studies also found no significant association between these two parameters (42-44).

Regarding the relationship between CAs and TL, Hemminki et al (17) previously demonstrated that shorter TL correlates with elevated levels of CAs in control individuals. In the present study, the same trend of significant negative correlation between ACs/CAtot/CSAs and TL in the entire group of controls was observed independently. It has been widely reported that telomeres progressively shorten with increased age. Initial TL can vary among individuals, where the rate of telomere shortening reflects replicative exhaustion during aging (45). In the absence of a telomere maintenance mechanism, cells eventually undergo replicative senescence (20). In the present group of control individuals, dependence of TL on age was found, where telomeres gradually shortened with advancing age. Age was also revealed to be associated with the accumulation of CSAs in control individuals. The association between this finding and the lower DNA repair rate in older subjects remains to be elucidated.

In the present study, it should be noted that apart from the correlation found between TL and age in control individuals, all the remaining statistically significant results should not be overinterpreted since the Spearman's $\rho$ values were small. It means that even though the relationships analyzed were statisti- cally significant, their biological relevance may remain limited. In a previous study, Bernadotte et al (45) proposed that instead of measuring the average TL, it would be reasonable to consider the quantity of shortened telomeres as an indicator of cellular and tissue proliferative potential. This is because cells do not exhaust their proliferative potentials in a simultaneous manner. Notably, patients with cancer did not exhibit any associations between TL and any type of CAs. Since there is no available literature that previously described this phenomenon, it can only be hypothesized that in complex and multifactorial diseases such as cancer, as yet unknown interactive pathways may exist that contribute to DNA damage and formation of CIN. Therefore, there is a need to conduct additional studies to elucidate this relationship between CAs and TL in the PBL of patients with cancer. We also did not record any clear correlation between TL and age in cancer patients. This was in contrast with the results obtained from the majority of previous studies covering this topic, which were summarized by Xu et al (46) and found that TL negatively correlated with age in patients with cancer.

Although CAs in PBL are proven markers of exposure, the role of this parameter in the pathophysiology and progression of cancer remain poorly understood (47). This uncertainty also applies for the prognostic value of TL in PBL for patients with cancer. The hypothesis that variations in CA frequencies and TL are determinants of prognosis is plausible to explain the heterogeneity in the clinical outcomes of patients with cancer. This therefore warrants further attention. In the present study, the prognostic power of CAs and TL was determined in all groups of patients with cancer. Association was not observed between any types of CAs and overall survival (OS) or recurrence-free survival (RFS) using univariate survival analysis. However, outputs obtained using the CART analysis revealed the involvement of CTAs in predicting the survival/mortality of patients, such that elevated CTAs was found to be a terminal node for lower OS for patients with $\mathrm{BC}$ at TNM stages $0+\mathrm{I}+\mathrm{II}+\mathrm{III}$ and for patients with CRC at TNM stage III. For CRC, an increase in CTAs $>1$ was found to worsen OS by $\sim 55 \%$. Of note, patients with $\mathrm{BC}$ without any CSAs exhibited inferior RFS. Based on these observations, different types of CAs may serve different roles in both cancer risk and patient prognosis. Numerous studies have previously explored the relationship between chromosomal rearrangements/CIN in PBL and the prognosis of patients with leukemia and lymphoma, the collective findings of which are summarized by Tanaka et al (48). However, no available accounts currently exist that explored such a relationship in solid tumors. Therefore, there remains a necessity in performing further extensive studies regarding this topic.

In terms of TL, no association could be found between telomere shortening and OS or RFS in the groups of patients with cancer, using either univariate or multivariate survival models. Several studies investigated the possible effects of TL on cancer prognosis, both in PBL and tumor tissues. A previous meta-analysis (46) of 29 studies revealed the association between shortened TL in blood and inferior OS and RFS in solid cancers, which is even stronger in hematological malignancies. The biological relevance of results on TL obtained from blood, and that obtained from target tissues remains unclear (49) PBL is indeed an available tissue that is suitable for repeated monitoring, which has a specific gatekeeping role in cancer, namely immunocompetency. 
Kroupa et al (50) recently demonstrated that patients with CRC containing smaller TL ratios between the tumor tissue and the adjacent cancer-free mucosa were associated with superior OS. By comparing the outputs obtained from both univariate and multivariate analyses among the individual groups of patients with cancer, survival in patients with LC was not affected by altering any of the genetic markers studied. Even the well-established parameter TNM stage was not found to influence survival. This was most likely due to the majority of patients with LC being diagnosed at advanced stages, mainly at TNM III + IV.

In summary, results from the present study suggest that increased frequency of ACs, CAtot, and CTAs in PBL may be considered as a marker of $\mathrm{BC}$ and $\mathrm{LC}$ risk. It was also observed that individuals with longer TL in PBL were at a higher risk of BC. Accumulation of CTAs in PBL was associated with worsened $\mathrm{OS}$ in patients with $\mathrm{BC}$ and $\mathrm{CRC}$ after stratification according to disease characteristics using a multivariate survival model. Neither the OS nor the RFS of the patients was found to be influenced by TL alterations. Notably, unlike those observed with control individuals, patients with cancer did not exhibit any associations between TL and CA frequencies or TL and age. The present study is associated with a number of limitations, particularly concerning the small sample sizes of individuals included, which served as one of the possible reasons for the reduced statistical power. In addition, outputs acquired using CART analysis should be treated with caution, since only individuals with full sets of data in all of the implied variables were included, which could have introduced selection bias. Nevertheless, the present study was performed based on validated and well-established methods with clearly defined clinical data.

In conclusion, increased frequency of chromosomal damage and TL in surrogate tissues, namely lymphocytes, which mediate antitumor immune responses, may to be applied to reflect cancer risk and progression. This underlines its potential applications in clinical oncology, which was confirmed by the rapidly rising number of similar studies. Therefore, additional studies with larger cohorts and consistent data on CAs and TL are warranted.

\section{Acknowledgements}

Not applicable.

\section{Funding}

The present study was funded by The Grant Agency of the Charles University (grant no. GAUK 800216 to SV), The Grant Agency of the Czech Republic (grant no. GACR 18-09709S to PV), Internal Grant Agency of the Ministry of Health of the Czech Republic (grant no. AZV 15-27580A to PV), Research program of Charles University, (grant no. PROGRES Q 28 Oncology to ZP, PV and LV), the project Biomedical Center Martin Slovakia co-financed from EU sources (grant no. ITMS 26220220187 to LM), The National Sustainability Program I (NPU I) provided by the Ministry of Education Youth and Sports of the Czech Republic (grant no. LO1503) and Charles University Research Centre program (grant no. UNCE/MED/006 'University Center of Clinical and Experimental Liver Surgery' to PV and LV).

\section{Availability of data and materials}

The datasets generated and/or analyzed during the present study are not publicly available to protect the personal identity of patients but are available from the corresponding author on reasonable request.

\section{Authors' contributions}

SV, KH, RaK and PV conceived the study. LM, MA and MS provided the hospital-based samples. ReK, LM and LV performed data collection of the patients. SV performed data curation. SV, ZP and LM performed chromosomal analysis. MK and SR performed the measurement of telomere length. SV performed data analyses and interpreted the results with assistance from MK, KH, RaK and PV. SV wrote and edited the manuscript. $\mathrm{ZP}, \mathrm{LM}, \mathrm{LV}, \mathrm{KH}, \mathrm{RaK}$ and PV revised the manuscript. ZP, RaK and PV supervised the project. LV administered the project. All authors read and approved the final version of the manuscript.

\section{Ethics approval and consent to participate}

The present study adhered to the ethical guidelines of the Helsinki Declaration. All participants were informed about all aspects of the study, agreed with the purpose of the study and procedures to be undertaken, following which they provided informed consent. The present study design was approved by the local Ethics Committees of all participating hospitals.

\section{Patient consent for publication}

Not applicable.

\section{Competing interests}

The authors declare that they have no competing interests.

\section{References}

1. Schvartzman JM, Sotillo R and Benezra R: Mitotic chromosomal instability and cancer: Mouse modelling of the human disease. Nat Rev Cancer 10: 102-115, 2010.

2. Hasty P and Montagna C: Chromosomal rearrangements in cancer: Detection and potential causal mechanisms. Mol Cell Oncol 1: e29904, 2014

3. Chatterjee A, Rodger EJ and Eccles MR: Epigenetic drivers of tumourigenesis and cancer metastasis. Semin Cancer Biol 51: $149-159,2018$

4. Obe G, Pfeiffer P, Savage JR, Johannes C, Goedecke W, Jeppesen P, Natarajan AT, Martínez-López W, Folle GA and Drets ME: Chromosomal aberrations: Formation, identification and distribution. Mutat Res 504: 17-36, 2002.

5. Natarajan AT and Palitti F: DNA repair and chromosomal alterations. Mutat Res 657: 3-7, 2008.

6. Bignold LP: Mechanisms of clastogen-induced chromosomal aberrations: A critical review and description of a model based on failures of tethering of DNA strand ends to strand-breaking enzymes. Mutat Res 681: 271-298, 2009.

7. Mateuca RA, Decordier I and Kirsch-Volders M: Cytogenetic methods in human biomonitoring: principles and uses. Methods Mol Biol 817: 305-334, 2012.

8. Musak L, Smerhovsky Z, Halasova E, Osina O, Letkova L, Vodickova L, Polakova V, Buchancova J, Hemminki K and Vodicka P: Chromosomal damage among medical staff occupationally exposed to volatile anesthetics, antineoplastic drugs, and formaldehyde. Scand J Work Environ Health 39: 618-630, 2013. 
9. Hagmar L, Stromberg U, Bonassi S, Hansteen IL, Knudsen LE, Lindholm $\mathrm{C}$ and Norppa $\mathrm{H}$ : Impact of types of lymphocyte chromosomal aberrations on human cancer risk: Results from Nordic and Italian cohorts. Cancer Res 64: 2258-2263, 2004.

10. Bonassi S, Norppa H, Ceppi M, Strömberg U, Vermeulen R, Znaor A, Cebulska-Wasilewska A, Fabianova E, Fucic A, Gundy S, et al: Chromosomal aberration frequency in lymphocytes predicts the risk of cancer: Results from a pooled cohort study of 22 358 subjects in 11 countries. Carcinogenesis 29: 1178-1183, 2008.

11. Vodicka P, Polivkova Z, Sytarova S, Demova H, Kucerova M, Vodickova L, Polakova V, Naccarati A, Smerhovsky Z, Ambrus M, et al: Chromosomal damage in peripheral blood lymphocytes of newly diagnosed cancer patients and healthy controls. Carcinogenesis 31: 1238-1241, 2010.

12. Vodenkova S, Polivkova Z, Musak L, Smerhovsky Z, Zoubkova H, Sytarova S, Kavcova E, Halasova E, Vodickova L, Jiraskova K, et al: Structural chromosomal aberrations as potential risk markers in incident cancer patients. Mutagenesis 30: 557-563, 2015.

13. Albertson DG, Collins C, McCormick F and Gray JW: Chromosome aberrations in solid tumors. Nat Genet 34: 369-376, 2003.

14. Boffetta P, van der Hel O, Norppa H, Fabianova E, Fucic A Gundy S, Lazutka J, Cebulska-Wasilewska A, Puskailerova D, Znaor A, et al: Chromosomal aberrations and cancer risk: Results of a cohort study from Central Europe. Am J Epidemiol 165: 36-43, 2007.

15. Li H, Hilmarsen HT, Hossain MB, Björk J, Hansteen IL, Albin M, Furu Skjelbred C and Broberg K: Telomere length and LINE1 methylation is associated with chromosomal aberrations in peripheral blood. Genes Chromosomes Cancer 52: 1-10, 2013.

16. Xu L, Li S and Stohr BA: The role of telomere biology in cancer. Annu Rev Pathol 8: 49-78, 2013.

17. Hemminki K, Rachakonda S, Musak L, Vymetalkova V, Halasova E, Försti A, Vodickova L, Buchancova J, Vodicka P and Kumar R: Telomere length in circulating lymphocytes: Association with chromosomal aberrations. Genes Chromosomes Cancer 54: 194-196, 2015.

18. Heidenreich B and Kumar R: TERT promoter mutations in telomere biology. Mutat Res 771: 15-31, 2017.

19. Maciejowski $\mathbf{J}$ and de Lange T: Telomeres in cancer: Tumour suppression and genome instability. Nat Rev Mol Cell Biol 18 : 175-186, 2017.

20. Shay JW: Role of telomeres and telomerase in aging and cancer. Cancer Discov 6: 584-593, 2016.

21. Ernst A, Jones DT, Maass KK, Rode A, Deeg KI, Jebaraj BM, Korshunov A, Hovestadt V, Tainsky MA, Pajtler KW, et al: Telomere dysfunction and chromothripsis. Int J Cancer 138 2905-2914, 2016

22. Rossner P, Cerna M, Bavorova H, Pastorkova A and Ocadlikova D Monitoring of human exposure to occupational genotoxicants. Cent Eur J Public Health 3: 219-223, 1995.

23. Cawthon RM: Telomere length measurement by a novel monochrome multiplex quantitative PCR method. Nucleic Acids Res 37: e21, 2009.

24. Heidenreich B, Rachakonda PS, Hosen I, Volz F, Hemminki K, Weyerbrock A and Kumar R: TERT promoter mutations and telomere length in adult malignant gliomas and recurrences. Oncotarget 6: 10617-10633, 2015.

25. Hosen I, Rachakonda PS, Heidenreich B, de Verdier PJ, Ryk C Steineck G, Hemminki K and Kumar R: Mutations in TERT promoter and FGFR3 and telomere length in bladder cancer. Int J Cancer 137: 1621-1629, 2015.

26. Kroupa M, Polivkova Z, Rachakonda S, Schneiderova M, Vodenkova S, Buchler T, Jiraskova K, Urbanova M, Vodickova L, Hemminki K, et al: Bleomycin-induced chromosomal damage and shortening of telomeres in peripheral blood lymphocytes of incident cancer patients. Genes Chromosomes Cancer 57: 61-69, 2018.

27. Lemon SC, Roy J, Clark MA, Friedmann PD and Rakowski W: Classification and regression tree analysis in public health: Methodological review and comparison with logistic regression. Ann Behav Med 26: 172-181, 2003.

28. Negrini S, Gorgoulis VG and Halazonetis TD: Genomic instability-an evolving hallmark of cancer. Nat Rev Mol Cell Biol 11: 220-228, 2010 .

29. Hanahan D and Weinberg RA: Hallmarks of cancer: The next generation. Cell 144: 646-674, 2011.

30. Gisselsson D: Chromosome instability in cancer: How, when, and why? Adv Cancer Res 87: 1-29, 2003
31. Gay-Bellile M, Romero P, Cayre A, Véronèse L, Privat M, Singh S, Combes P, Kwiatkowski F, Abrial C, Bignon YJ, et al: ERCC1 and telomere status in breast tumours treated with neoadjuvant chemotherapy and their association with patient prognosis. J Pathol Clin Res 2: 234-246, 2016.

32. Gardner M, Bann D, Wiley L, Cooper R, Hardy R, Nitsch D, Martin-Ruiz C, Shiels P, Sayer AA, Barbieri M, et al: Gender and telomere length: systematic review and meta-analysis. Exp Gerontol 51: 15-27, 2014

33. Mayer S, Bruderlein S, Perner S, Waibel I, Holdenried A, Ciloglu N, Hasel C, Mattfeldt T, Nielsen KV and Möller P: Sex-specific telomere length profiles and age-dependent erosion dynamics of individual chromosome arms in humans. Cytogenet Genome Res 112: 194-201, 2006.

34. Nawrot TS, Staessen JA, Gardner JP and Aviv A: Telomere length and possible link to X chromosome. Lancet 363: 507-510, 2004.

35. Kyo S, Takakura M, Kanaya T, Zhuo W, Fujimoto K, Nishio Y, Orimo A and Inoue M: Estrogen activates telomerase. Cancer Res 59: 5917-5921, 1999.

36. Misiti S, Nanni S, Fontemaggi G, Cong YS, Wen J, Hirte HW, Piaggio G, Sacchi A, Pontecorvi A, Bacchetti S and Farsetti A: Induction of hTERT expression and telomerase activity by estrogens in human ovary epithelium cells. Mol Cell Biol 20: 3764-3771, 2000 .

37. Svenson U, Nordfjall K, Stegmayr B, Manjer J, Nilsson P, Tavelin B, Henriksson R, Lenner P and Roos G: Breast cancer survival is associated with telomere length in peripheral blood cells. Cancer Res 68: 3618-3623, 2008.

38. Gramatges MM, Telli ML, Balise R and Ford JM: Longer relative telomere length in blood from women with sporadic and familial breast cancer compared with healthy controls. Cancer Epidemiol Biomarkers Prev 19: 605-613, 2010.

39. Levy T, Agoulnik I, Atkinson EN, Tong XW, Gause HM, Hasenburg A, Runnebaum IB, Stickeler E, Möbus VJ, Kaplan AL and Kieback DG: Telomere length in human white blood cells remains constant with age and is shorter in breast cancer patients. Anticancer Res 18: 1345-1349, 1998.

40. Pooley KA, Sandhu MS, Tyrer J, Shah M, Driver KE, Luben RN, Bingham SA, Ponder BA, Pharoah PD, Khaw KT, et al: Telomere length in prospective and retrospective cancer case-control studies. Cancer Res 70: 3170-3176, 2010.

41. Qu S, Wen W, Shu XO, Chow WH, Xiang YB, Wu J, Ji BT, Rothman N, Yang G, Cai Q, et al: Association of leukocyte telomere length with breast cancer risk: Nested case-control findings from the Shanghai Women's Health Study. Am J Epidemiol 177: 617-624, 2013.

42. Shen J, Terry MB, Gurvich I, Liao Y, Senie RT and Santella RM: Short telomere length and breast cancer risk: A study in sister sets. Cancer Res 67: 5538-5544, 2007.

43. Shen J, Gammon MD, Terry MB, Wang Q, Bradshaw P, Teitelbaum SL, Neugut AI and Santella RM: Telomere length, oxidative damage, antioxidants and breast cancer risk. Int $\mathrm{J}$ Cancer 124: 1637-1643, 2009.

44. Zheng YL, Ambrosone C, Byrne C, Davis W, Nesline M and McCann SE: Telomere length in blood cells and breast cancer risk: Investigations in two case-control studies. Breast Cancer Res Treat 120: 769-775, 2010.

45. Bernadotte A, Mikhelson VM and Spivak IM: Markers of cellular senescence. Telomere shortening as a marker of cellular senescence. Aging (Albany NY) 8: 3-11, 2016.

46. Xu X, Qu K, Pang Q, Wang Z, Zhou Y and Liu C: Association between telomere length and survival in cancer patients: A meta-analysis and review of literature. Front Med 10: 191-203, 2016.

47. Venkatesan S, Natarajan AT and Hande MP: Chromosomal instability-mechanisms and consequences. Mutat Res Genet Toxicol Environ Mutagen 793: 176-184, 2015.

48. Tanaka K and Hirota T: Chromosomal instability: A common feature and a therapeutic target of cancer. Biochim Biophys Acta 1866: 64-75, 2016

49. Ennour-Idrissi K, Maunsell E and Diorio C: Telomere length and breast cancer prognosis: A systematic review. Cancer Epidemiol Biomarkers Prev 26: 3-10, 2017.

50. Kroupa M, Rachakonda SK, Liska V, Srinivas N, Urbanova M, Jiraskova K, Schneiderova M, Vycital O, Vymetalkova V, Vodickova L, et al: Relationship of telomere length in colorectal cancer patients with cancer phenotype and patient prognosis. $\mathrm{Br}$ J Cancer 121: 344-350, 2019. 